\title{
Ranking Opportunity Profiles through Dependent Evaluation of Policies *
}

\author{
Jorge Alcalde-Unzu ${ }^{1} \quad$ Miguel A. Ballester ${ }^{2}$
}

January 2011

\begin{abstract}
${ }^{1}$ Corresponding author. Department of Economics, Public University of Navarre. Campus Arrosadia, 31006, Pamplona, Spain. E-mail: jorge.alcalde@unavarra.es

2 Departament d'Economia i d'Història Econòmica, Universitat Autònoma de Barcelona, Bellaterra, Barcelona, 08193, Spain. E-mail: miguelangel.ballester@uab.es
\end{abstract}

${ }^{*}$ We are very grateful to John Weymark for his highly valuable comments and suggestions. We would also like to thank Amaia Arandia, Ritxar Arlegi, Juan R. de Miguel, Laurence Kranich, Jorge Nieto and Yongsheng Xu for their comments. Financial support from the Spanish Ministry of Education through grants ECO2008-04756, ECO200911213, ECO2009-12836, Juan de la Cierva and Ramon y Cajal programs, FEDER, and the Barcelona Economics Program of CREA is gratefully acknowledged. 


\begin{abstract}
Rankings to evaluate opportunity distributions present in most literature judge a policy (change from one distribution of opportunities to another) on the basis of the changes created and, thus, independently of the original situation. This paper proposes a group of axioms capturing the idea that rankings of equality of opportunities might consider not only the changes promoted, but also the initial situation in society. The combination of this group of axioms with other well-established properties enables us to characterize two families of new opportunity distribution rankings. The first family weighs each individual's percentage share in the total number of opportunities, while the second weighs opportunities depending on how many agents have them available.
\end{abstract}

Keywords and Phrases: Opportunity profiles, Advantage, Equality, Evaluation of Policies. 


\section{Introduction}

\section{Opportunities}

According to classical theories of welfarism, social situations should be evaluated by the extent to which the preferences of individuals are satisfied. Hence, in line with the view that individuals choose the life providing them with the greatest welfare, we could merely evaluate social situations by observing the way of life actually chosen by individuals. ${ }^{1}$

However, several contemporary theories of justice are less enthusiastic on such a narrow way of judging societies. Examples include Dworkin [8, 9], who proposes paying attention to individual resources when describing the goodness of a social situation. Rawls [21] places special emphasis on certain resources available to agents, i.e., primary goods. Sen [23] complements Rawls' approach by suggesting that capabilities to function be considered, i.e., the interaction between available resources and individual abilities. Arneson [3] also suggests departing from actual welfare and focus on opportunity for welfare.

In a nutshell, some of these authors argue that preferences can be affected by the availability of opportunities, as in the case of adaptive preferences, posed by Elster [11]. These theories further endorse that the intrinsic value of the availability of opportunities, i.e., the freedom of choice, has to be considered. ${ }^{2}$ Hence, we should pay more attention to an objective description of the set of opportunities available to individuals at the moment of taking life decisions, while incorporating the intrinsic value of the availability of opportunities. In short, social situations should be judged by describing, for each individual, the collection of alternative lives available: their opportunity sets.

\footnotetext{
${ }^{1}$ In practice, most studies tend to focus exclusively on income as a proxy for welfare.

${ }^{2}$ Sen $[23,24]$ and Pattanaik and Xu [19] also discuss the role of preferences vis-a-vis opportunities.
} 
From a more technical perspective, an available life or opportunity can be understood as a description of all the aspects of a possible life the individual may choose to follow. There are at least two different formalizations of this concept that have attracted attention. In the literature that goes back to the measurement of freedom of choice, an opportunity is an abstract and flexible description of those aspects of life that we may consider relevant. Thus, opportunities have no particular mathematical structure and an opportunity set is just a subset of the universal set of opportunities. ${ }^{3}$ In the literature that goes back to the notion of capabilities, an opportunity is a vector of functionings, where functionings are all kinds of personal achievements that individuals may obtain in their lives. Then, an opportunity set can be represented by a set (not necessarily convex) in the space $\mathbb{R}^{n}{ }^{4}$ Not surprisingly, these two literatures are intimately connected. In many cases, the latter constitutes a particularization of the former that allows those nonwelfarist approaches to be used in applied studies. An example is the study of Echavarri and Permanyer [10] that adapts the proposals of Herrero et al. [14] to the context of functionings.

In this paper, we adopt the view that social situations should be ranked considering the opportunity sets available to individuals in society objectively. We formalize our debate technically by using the more flexible modelization of opportunities.

\section{Evaluation of Opportunity Distributions}

A natural approach to ranking social situations in terms of opportunity sets of individuals is to incorporate the key classical concepts of inequality and,

\footnotetext{
${ }^{3}$ See, for instance, Kranich [16], Herrero [13], Herrero et al. [14], Bossert et al. [6], Ok and Kranich [18], Savaglio and Vanucci [22], Weymark [26], Alcalde-Unzu et al. [1] and Alcalde-Unzu and Ballester [2] (for a survey of some of this literature, see Peragine [20]).

${ }^{4}$ In this context, opportunity sets are also called capability sets. See, for instance, Fleurbaey [12] and Echavarri and Permanyer [10].
} 
as a consequence, to reformulate the notion of advantage embedded in them. For instance, the Pigou-Dalton transfer principle states that a transfer from an advantaged to a disadvantaged individual (without reversing the order) results in a better social situation. In the income distribution framework, the notion of advantage is defined through the well-ordered welfare or income of individuals. However, the non-welfarist framework of opportunities lacks a unique clear notion of advantage. A widely used candidate to substitute income is the cardinality of the opportunity set (see Pattanaik and $\mathrm{Xu}[19])$. This ranking can be considered to only measure very limited aspects of freedom of choice and it is thus a rather crude basis for comparing individual opportunity sets. However, according to the view that individual preferences are not as relevant, few alternatives remain. We may obviously use a social common ranking for evaluating the goodness of an opportunity set. Unfortunately, Ok [17] proved that the cardinality-based criterion is the unique complete ranking that makes the reformulation of the Pigou-Dalton transfer principle and some other basic properties of equality compatible. An incomplete ranking of individual opportunity sets may alternatively be considered. Existing literature has paid special attention to the inclusion ranking (that is, an individual is advantaged with respect to another only if the former has all the opportunities available to the latter).

Alcalde-Unzu and Ballester [2] present characterization results showing that most of the literature on ranking social situations through opportunity sets can be summarized by accepting either the cardinality or the inclusion notion of advantage and building upon one of these notions by:

(a) Imposing minimal conditions regarding the goodness of opportunity distributions based on some classical fairness concepts: Anonymity, which implies that the names of the agents should not matter; Assimilation, which implies that the opportunity profile obtained as a result of the addition of common opportunities to all agents or the replacement of distinct opportu- 
nities by common ones, should not be worse than the original opportunity profile; and Priority for the Poor, which implies that giving new opportunities to those agents who are undoubtedly most disadvantaged should be better than giving them to other agents (or to no agent). ${ }^{5}$

(b) Imposing an Independence condition on the policies undertaken resulting in changes in the sets of opportunities available to different agents in society. ${ }^{6}$ Specifically, the comparison of the goodness of two societies is not modified by the application of a common policy. Together with the properties in (a), Independence implies that policies are evaluated either as positive or negative, but this evaluation is independent of the initial distribution of opportunities.

This paper seeks to promote the idea that the initial situation should matter when evaluating policies that change the distribution of opportunities. For example, perturbing a perfectly equal society to obtain some extra opportunities might be perceived as substantially different in a society where individuals already have a wide variety of opportunities or in another where they have few opportunities. Consequently, we look for criteria to rank social situations that accept either the cardinality or the inclusion notion of advantage and build upon one of these notions combining the three well-established goodness properties of part (a) with some axioms that capture the idea that the goodness of a policy should depend on the original situation of society. As a result, Theorems 3.1 and 3.2 analyze how to reconsider the classical concept of equality of opportunities, suggesting that social situations should be judged as follows.

Under the cardinality approach, Theorem 3.1 establishes a family of

\footnotetext{
${ }^{5}$ Priority for the Poor is called Monotonicity in Alcalde-Unzu and Ballester [2].

${ }^{6}$ The technical definition of a policy will be provided in Section 2. In non-technical terms, a policy is a description of the differences between any two opportunity profiles. These differences could eventually (but not necessarily) be the result of the application of a policy undertaken by a government to the first profile leading to the second profile.
} 
criteria that weighs each individual's percentage share in the total number of opportunities. These weights decrease with respect to the cardinality of the opportunity set, in the sense that individuals with fewer opportunities are assigned a higher weight in the social comparison. Under the partial inclusion approach, Theorem 3.2 establishes a family of rankings that weighs opportunities depending on how many agents have them available. The weights decrease with respect to this number of individuals, in the sense that the percentage of opportunities shared by all individuals has greater weight than the percentage of opportunities shared by all the individuals except one, and so on.

The remainder of this paper is divided as follows. Section 2 discusses the existing criteria to rank social situations in terms of opportunities. Section 3 introduces properties that allow the goodness of policies to depend on the initial social situation and includes the new characterization results. Section 4 concludes. Finally, the proofs of the results are included in the Appendix.

\section{Ranking Opportunity Distributions through In- dependent Evaluation of Policies}

Our stylized model deals mainly with the three-agent case. We consider this case to be the most prominent, as it preserves the clarity and intuition of the common two-agent case within this literature, while it also incorporates some interesting features that are only present in the general ( $n$-agent) case. For instance, in a three-agent society, it is possible to consider how an agent who is neither the most disadvantaged nor the most advantaged should be treated in the pursuit of equity. Hence, consider a society consisting of three individuals $I=\{1,2,3\}$ and an infinite set $X$ that describes all possible opportunities. An opportunity set for agent $i \in I$ is an element $O_{i} \in L$, where $L$ denotes the set of all non-empty and finite subsets of $X$. 
A social situation will be judged according to the profile of opportunity sets $O=\left(O_{1}, O_{2}, O_{3}\right) \in L^{3}$. We look for criteria to rank profiles of opportunity sets, and we denote by $\succsim \subseteq L^{3} \times L^{3}$ a transitive and complete binary relation. We interpret $O \succsim U$ as "profile $\mathrm{O}$ is socially preferred to profile $\mathrm{U}$ ". The relations $\succ$ and $\sim$ are defined as usual.

Given $O \in L^{3}$, we say that the collection of sets $p=\left\{A_{i}, B_{i}\right\}_{i \in I} \in(L \cup \emptyset)^{6}$ such that $A_{i} \subseteq O_{i}, B_{i} \cap O_{i}=\emptyset$ and $O_{i}^{p}=\left(O_{i} \backslash A_{i}\right) \cup B_{i} \in L$ for all $i \in I$ is a policy compatible with $O$. A compatible policy describes the opportunities that are added to and removed from the opportunity sets of the individuals in the society. That is, given the initial situation of individual $i$ described by opportunity set $O_{i}$, opportunities $A_{i}$ are removed from the opportunity set of individual $i$, and opportunities $B_{i}$ are added to the opportunity set of individual $i .^{7}$ As a consequence of the removal and addition of opportunities, individual $i$ resulting set of opportunities $O_{i}^{p}$ should be non-empty. We say that the opportunity profile $O^{p}=\left(O_{1}^{p}, O_{2}^{p}, O_{3}^{p}\right)$ is the social consequence of applying the policy $p$ to the profile $O$. We say that a policy $p$ applied to a profile $O \in L^{3}$ is good (respectively bad, respectively neutral) if the profile $O^{p}$ obtained after the application of the policy $p$ is strictly better (respectively strictly worse, respectively indifferent) than the initial profile $O$.

The first three basic properties of the model judge certain policies as good, bad or neutral policies. The first classical property on the evaluation of social situations is that the names of the agents should not matter. To formally define this property, we make use of some particular permutations over the set of individuals. Given a social situation $O \in L^{3}, \sigma^{O}$ denotes the set of permutations $\sigma$ of $I$ such that $\left|O_{\sigma(3)}\right| \leq\left|O_{\sigma(2)}\right| \leq\left|O_{\sigma(1)}\right|$. The profile $\left(O_{\sigma(1)}, O_{\sigma(2)}, O_{\sigma(3)}\right)$ is denoted by $\sigma(O) .^{8}$

\footnotetext{
${ }^{7}$ The requirements $A_{i} \subseteq O_{i}$ and $B_{i} \cap O_{i}=\emptyset$ are introduced to guarantee that the impact of a policy is equivalent across different profiles.

${ }^{8}$ Throughout the paper, we avoid making the universal quantifier for permutations
} 
Anonymity (ANON): $O \sim \sigma(O)$ for all $O \in L^{3}$.

In other words, a policy that reallocates the entire opportunity sets among the individuals is neutral. This is a standard property in the literature.

The second basic property deals with the provision of common opportunities to agents, which should be considered to be not worse than the provision of heterogeneous opportunities or the absence of any provision. To formally define this property, we introduce the following notation: $O^{\cup}$ will be the set that includes all opportunities that at least one individual in $O$ has; i.e, $O^{\cup}=\bigcup_{i \in I} O_{i}$ and, similarly, $O^{\cap}$ will be the set of opportunities that all individuals in $O$ have; i.e, $O^{\cap}=\bigcap_{i \in I} O_{i}$.

Assimilation (ASM): For all $O \in L^{3}$ and for all $x \in\left(X \backslash O^{\cup}\right)$ and $y_{1}, y_{2}, y_{3} \in X,\left(O_{1} \cup\{x\}, O_{2} \cup\{x\}, O_{3} \cup\{x\}\right) \succsim\left(O_{1} \cup\left\{y_{1}\right\}, O_{2} \cup\left\{y_{2}\right\}, O_{3} \cup\left\{y_{3}\right\}\right)$ whenever:

1. $y_{i} \in O_{i}$ for all $i \in I$ or

2. for all $K \subseteq I$, there exists $y \in\left\{y_{k}\right\}_{k \in K}$ such that $y \notin \bigcup_{k \in K} O_{k}$.

In other words, the first part of the property says that the policy that assigns a novel common opportunity to all individuals is never bad. This first part is a weakening of the standard Independence of Common Expansions property that imposes indifference between the original situation and the resulting one. The second part of the property says that a policy removing a certain uncommon opportunity of each agent and providing a common opportunity to all of them is never bad. This second part is a weakening of the standard Assimilation property to avoid certain controversies. For a wide discussion on these weakenings, see Alcalde-Unzu and Ballester [2]. The justification of this property differs depending on the notion of advantage explicit when writing $\sigma(O)$. 
adopted. If the cardinality-based criterion is used to judge advantage, this implies indirectly that the quality of all opportunities is equivalent and the axiom is then not controversial. If, however, we have adopted the Partial Inclusion Ranking, there is no information on the quality of the opportunities. The addition of the same opportunity to both profiles does not add new inequalities between the individuals. On the other hand, the addition of different opportunities may decrease or increase the degree of equality of the profile depending on the previous relative situation of the individuals and the possibly different quality of the new opportunities. This is totally unknown as the partial inclusion criterion has been adopted. What the axiom is imposing in this case is that the criterion to rank opportunity profiles should not be risk lover in this context of complete uncertainty, something that the majority of criteria proposed in the literature of complete uncertainty satisfies (two classical examples are the maxmin and the protective criteria. See Barbera and Jackson [5]).

The third basic property has to do with a basic idea of equalization. Giving new opportunities to those agents who are undoubtedly most disadvantaged should be better than giving them to other agents or to no agent. To formally define this property, we make use of the concept of nested profiles of opportunity sets. A profile $O$ is nested if $O_{\sigma(3)} \subseteq O_{\sigma(2)} \subseteq O_{\sigma(1)}$ for all $\sigma \in \sigma^{O}$. In particular, $\mathcal{N}$ denotes the set of nested profiles in which the identity mapping belongs to $\sigma^{O}$. That is, $\mathcal{N}=\left\{O \in L^{3}\right.$ such that $O_{3} \subseteq$ $\left.O_{2} \subseteq O_{1}\right\}$. We also define for any $O \in L^{3}, i \in I$ and $x \in X$, a new profile $\bar{O}(x, i)$ for which individual $i$ has the set $O_{i} \cup\{x\}$ and any other individual $j \in I \backslash\{i\}$ has the opportunity set $O_{j}$.

Priority for the Poor (PRI): For all $O \in \mathcal{N}$, for all $i, j \in I$ such that $j>i$ and for all $x \notin O_{j}, \bar{O}(x, j) \succsim \bar{O}(x, i)$, with strict preference if $j=3$ and $x \in O_{k}$ for all $k \neq 3$. 
In other words, we can undoubtedly claim in a nested profile that agent 1 is more advantaged than agent 2 and also, agent 2 is more advantaged than agent $3 .{ }^{9}$ Hence, any policy that assigns an opportunity to a disadvantaged individual is never bad. Any policy that removes an opportunity from an advantaged individual and assigns it to a disadvantaged individual is never bad. Finally, a policy that assigns an opportunity to the most disadvantaged individual that all the other individuals already have is always good.

In addition to these properties, the proposals of the literature satisfy a condition of Independence that reflects the following idea: the application of a common policy with certain characteristics to two different societies does not modify the judgement regarding which of these societies is better. To introduce the axiom formally, some extra notation is needed. A nonreversal policy is a policy where the positions of the individuals according to the ranking used to judge advantage do not revert. ${ }^{10}$ As has already been stated, two possible rankings are studied: the Cardinality-based Criterion and the Partial Inclusion Criterion. Therefore, given $O \in \mathcal{N}$ a policy $p$ compatible with $O$ is a non-reversal policy according to the Cardinalitybased Criterion if $\left|O_{j}^{p}\right| \geq\left|O_{i}^{p}\right|$ for all $j<i$. Similarly, given $O \in \mathcal{N}$, a policy $p$ compatible with $O$ is a non-reversal policy according to the Partial Inclusion Criterion if $O_{j}^{p} \subset O_{i}^{p}$ does not occur for any $j<i$. Denote the set of non-reversal policies of $O$ according to the Cardinality-based Criterion by $\mathcal{P}_{O}^{\#}$ and the set of non-reversal policies of $O$ according to the Partial Inclusion Criterion by $\mathcal{P} \subseteq$. Depending on the ranking, we have two possible

\footnotetext{
${ }^{9}$ Note that this claim is true both for the set inclusion notion of advantage and the cardinality notion, which is merely a completion of the former.

${ }^{10}$ Non-reversal policies are also discussed in other frameworks, such as the literature on horizontal equity of tax policies. The idea behind horizontal equity is to describe a progressive transfer in which individuals are equalized, but their relative situation is not reverted. In tax literature, the pre-tax income distribution is considered to have ethical status that should not be totally violated (see King [15], for example).
} 
specifications of the axiom:

Independence-cardinality (IND-\#): For all $O, U \in \mathcal{N}$ and for all $p \in$ $\mathcal{P}_{O}^{\#} \cap \mathcal{P}_{U}^{\#}, O \succsim U \Rightarrow O^{p} \succsim U^{p}$

Independence-inclusion (IND- $\subseteq$ ): For all $O, U \in \mathcal{N}$ and for all $p \in$ $\mathcal{P} \stackrel{\subseteq}{\subseteq} \cap \mathcal{P} \underset{U}{\subseteq}, O \succsim U \Rightarrow O^{p} \succsim U^{p}$.

The combination of Independence and the above properties implies that any policy should be considered either good or bad, but judgement should always be made independently of the original society to which it is applied. The criteria of the literature differ in the specifications of the sets of good and bad policies. The following two theorems, included in Alcalde-Unzu and Ballester [2] provide the basic structure of all possible criteria satisfying the proposed properties. Before presenting them, we need to introduce an additional piece of notation: $\bigcap_{k} O=\{x \in X$ such that there exists $K \subseteq$ $I$ with $|K|=k$ and $x \in O_{i}$ for all $\left.i \in K\right\}$. Obviously, $\bigcap_{3} O=O^{\cap}$ and $\bigcap_{1} O=O^{\cup}$

Theorem 2.1 Let $\succsim$ be a criterion satisfying ANON, ASM, PRI and IND\#. Then, there exists $\alpha=\left(\alpha_{1}, \alpha_{2}\right)$ with $\alpha_{1} \leq \alpha_{2} \leq 1$ and $\alpha_{1}+\alpha_{2} \geq-1$ such that for all $O, U \in L^{3}$,

$$
\left|O_{\sigma(3)}\right|+\alpha_{2}\left|O_{\sigma(2)}\right|+\alpha_{1}\left|O_{\sigma(1)}\right|>\left|U_{\sigma(3)}\right|+\alpha_{2}\left|U_{\sigma(2)}\right|+\alpha_{1}\left|U_{\sigma(1)}\right| \Rightarrow O \succ U .
$$

Theorem 2.2 Let $\succsim$ be a criterion satisfying ANON, ASM, PRI and IND$\subseteq$. Then, there exists $\beta=\left(\beta_{1}, \beta_{2}\right)$ with $\beta_{1} \leq \beta_{2} \leq 1$ and $\beta_{1}+\beta_{2} \geq-1$ such that for all $O, U \in L^{3}$,

$$
\left|\bigcap_{3} O\right|+\beta_{2}\left|\bigcap_{2} O\right|+\beta_{1}\left|\bigcap_{1} O\right|>\left|\bigcap_{3} U\right|+\beta_{2}\left|\bigcap_{2} U\right|+\beta_{1}\left|\bigcap_{1} U\right| \Rightarrow O \succ U .
$$


The criteria of these two families differ in the values of the parameters $\alpha$ and $\beta$. These values reflect the different concerns for equality and efficiency issues of the criterion under consideration. To give one example, the criterion with $\alpha=(-1,0)$ is a criterion of the equality criteria family characterized by Kranich [16], meanwhile the criterion with $\alpha=(0,0)$ corresponding with the maxmin criterion (discussed by Bossert et al. [6]) shows more concern for efficiency issues. ${ }^{11}$

\section{Ranking Opportunity Distributions through De- pendent Evaluation of Policies}

Section 2 discusses rankings of opportunity distributions that incorporate the idea that the initial situation should not matter when evaluating policies that change the distribution of opportunities. To the best of our knowledge, this paper is the first attempt at promoting the idea that policies should be considered good or bad conditional on the society to which they are applied. Although this dependence may take many different forms, two properties are proposed in this section: Policy Monotonicity and Intermediateness, capturing the scope and degree of the idea of dependence of the status quo for a ranking of equality of opportunities. ${ }^{12}$ These axioms will express the idea that this dependence has to be produced with an idea of inequality aversion.

To motivate Policy Monotonicity, suppose that there exist two different societies $O$ and $U$, the first of which is considered to provide more equality of opportunities to individuals. Consider a policy that equalizes the indi-

\footnotetext{
${ }^{11}$ A fuller description of many of the criteria included in these two families and their characteristics in terms of equality and efficiency considerations is included in AlcaldeUnzu and Ballester [2].

${ }^{12}$ Given that the majority of the literature has focused on equality criteria, we have adopted this interpretation as a starting point for this literature.
} 
viduals in society $O$. Then, Policy Monotonicity states that the same policy equalizes also the individuals in the more unequal society $U$. Consider, for instance, that the policy consists of giving new opportunities to all agents and is able to improve the equality of $O$. That means that the opportunities given by the policy to the disadvantaged agents in society are sufficient to socially compensate the additional opportunities that the advantaged agents are receiving. It is intuitive to think that such a policy must also increase the equality (or, at least, not to decrease it) if applied to a more unequal society $U$, where the margin to improve equality is higher. Notice that Policy Monotonicity says nothing about the effects on $O$ of a policy that constitutes equalization for the more unequal society $U$.

Analogously, Policy Monotonicity also imposes that if a policy reduces the equality of society $U$ then it also reduces the equality of the more egalitarian society $O$. Additionally, the property also imposes that the application of the same policy to two equivalent or indifferent societies has the same effect on them. We propose two different formulations of the property, depending on the criterion used to evaluate advantage.

Policy Monotonicity-cardinality (PM-\#): For all $O, U \in \mathcal{N}$ and for all $p \in \mathcal{P}_{O}^{\#} \cap \mathcal{P}_{U}^{\#}:$

if $O \succ U$, then $\left\{\left[O^{p} \succsim O \Rightarrow U^{p} \succsim U\right]\right.$ and $\left.\left[U \succsim U^{p} \Rightarrow O \succsim O^{p}\right]\right\}$ and

if $O \sim U$, then $O^{p} \sim U^{p}$.

Policy Monotonicity-inclusion (PM- $\subseteq$ ): For all $O, U \in \mathcal{N}$ and for all $p \in \mathcal{P} \subseteq \cap \mathcal{P}_{\bar{U}}^{\subseteq}$ :

if $O \succ U$, then $\left\{\left[O^{p} \succsim O \Rightarrow U^{p} \succsim U\right]\right.$ and $\left.\left[U \succsim U^{p} \Rightarrow O \succsim O^{p}\right]\right\}$ and

if $O \sim U$, then $O^{p} \sim U^{p}$.

The second property, Intermediateness, requires the union of two social distributions to be ranked between the two. That is, the addition to a profile of a more (respectively, less) egalitarian profile constitutes a strict 
increase (respectively, reduction) in equality. If we interpret the union of two nested profiles $O$ and $U$ as the application of the "policy" $U$ to the profile $O$ (or, alternatively, the "policy" $O$ to the profile $U$ ), the axiom has other interpretation: a policy is good (respectively, bad) if, when it can be interpreted as a profile, the policy is more (respectively, less) egalitarian than the status quo distribution. Consider, for example, that $O$ is a perfectly egalitarian profile and $U$ is any profile with some inequality. Then, the union of $O$ and $U$ will have some inequality and, then, it will be ranked in terms of equality below $O$. However, it is natural to think that this joint profile will have less inequality than the profile $U$.

Intermediateness (INT): For all $O, U \in \mathcal{N}$ such that $O^{\cup} \cap U^{\cup}=\emptyset$,

$$
O \succ U \Rightarrow O \succ(O \cup U) \succ U
$$

We describe the structure of the criteria that satisfy these two new properties on top of the three classical properties described above. We need to introduce the following notation: for all $O \in L^{3}, q_{j}(O)=\frac{\left|O_{\sigma(j)}\right|}{\sum_{i=1}^{3}\left|O_{i}\right|}$ and $p_{j}(O)=\frac{\left|\bigcap_{j} O\right|}{\sum_{i=1}^{3}\left|O_{i}\right|}$ for all $j \in I$.

Theorem 3.1 If $\succsim$ satisfies ANON, ASM, PRI, PM-\# and INT, then there exists $\alpha \in[0,1]$ such that for all $O, U \in L^{3}$,

$$
q_{3}(O)+\alpha q_{2}(O)>q_{3}(U)+\alpha q_{2}(U) \Rightarrow O \succ U .
$$

Theorem 3.2 If $\succsim$ satisfies ANON, ASM, PRI, PM- $\subseteq$ and INT, then there exists $\beta \in[0,1]$ such that for all $O, U \in L^{3}$,

$$
p_{3}(O)+\beta p_{2}(O)>p_{3}(U)+\beta p_{2}(U) \Rightarrow O \succ U .
$$

Observe that when the Cardinality-based Criterion is used to rank individuals in terms of advantage, Theorem 3.1 establishes a family of criteria that weighs each individual's percentage share in the total number of 
opportunities. These weights decrease in these percentages, in the sense that individuals with fewer opportunities are assigned a higher weight in the social comparison. The value of the parameter $\alpha$ reflects the relative importance that the criterion gives to the individual in the median of the distribution (higher values indicate more importance). On the other hand, when the Partial Inclusion Ranking is used to evaluate advantage, Theorem 3.2 establishes a family of criteria that weighs the percentages of opportunities belonging to each intersection between the opportunity sets of the individuals. The weights of these intersection sets decrease in the number of individuals that share each opportunity, in the sense that the opportunities within the opportunity sets of all individuals have greater weight that the opportunities belonging to only two individual opportunity sets, and so on.

It is interesting to point out the role of the axioms in our results in comparison with the results of the independent case. In the independent evaluation of policies, we can think of the space of policies and determine the hyperplane that separates good policies from bad policies. IND plays there a crucial role in determining the existence of such hyperplane, while the basic ethical axioms (ANON, ASM and PRI) determine the type of hyperplanes to be found. In the dependent evaluation of policies, we can no longer proceed in this simple way, as a policy is going to be bad or good depending on the original opportunity profile. However, INT plays a crucial role for showing that for every social situation, a hyperplane divides the good and bad policies. PM is crucial to show that hyperplanes associated to different social situations have the same slope. The basic ethical axioms (ANON, ASM and PRI) determine again the type of hyperplanes to be found.

The results established in Theorems 3.1 and 3.2 are not complete characterizations of the criteria that satisfy the axioms. Consider the following additional Archimedean Difference property of Kranich [16] and Weymark 
[26] that establishes the following idea: If we have two infinite sequences of opportunity profiles such that each opportunity profile of the first is more egalitarian than its corresponding opportunity profile of the second, then we can compensate any large difference between any two opportunity profiles by adding a sufficiently large number of opportunity profiles of the sequences to each.

Archimedean Difference (ARCHD): Let $\left\{O^{k}\right\}_{k \in \mathbb{N}}$ and $\left\{U^{k}\right\}_{k \in \mathbb{N}}$ be two infinite sequences in $\mathcal{N}$ such that $O_{i}^{s} \cap O_{i}^{t}=U_{i}^{s} \cap U_{i}^{t}=\emptyset$ for all $s \neq t$ and all $i \in I$. If $O^{k} \succ U^{k}$ for all $k \in \mathbb{N}$, then for all $\hat{O}, \hat{U} \in \mathcal{N}$ there exists $K \in \mathbb{N}$ such that $O^{1} \cup O^{2} \cup \cdots \cup O^{K} \cup \hat{O} \succsim U^{1} \cup U^{2} \cup \cdots \cup U^{K} \cup \hat{U}$.

Archimedean Difference imposes that when fixing a pair of profiles $\hat{O}, \hat{U}$, regardless of the extent to which $\hat{U}$ might be more egalitarian than $\hat{O}$, this difference is eventually overshadowed by the disparities between $O^{1}, \ldots, O^{K}$ and $U^{1}, \ldots, U^{K}$ for sufficiently large $K$. In other words, there is no disparity that cannot be reversed by accumulating a sufficiently large number of other disparities.

Imposing ARCHD immediately implies, for each of the families, that all cases with an equal weighted sum are indifferent.

Corollary $3.1 \succsim$ satisfies ANON, ASM, PRI, PM-\#, INT and ARCHD if and only if there exists $\alpha \in[0,1]$ such that for all $O, U \in L^{3}$,

$$
q_{3}(O)+\alpha q_{2}(O) \geq q_{3}(U)+\alpha q_{2}(U) \Leftrightarrow O \succsim U .
$$

Corollary $3.2 \succsim$ satisfies ANON, ASM, PRI, PM- $\subseteq$, INT and ARCHD if and only if there exists $\beta \in[0,1]$ such that for all $O, U \in L^{3}$,

$$
p_{3}(O)+\beta p_{2}(O) \geq p_{3}(U)+\beta p_{2}(U) \Leftrightarrow O \succsim U .
$$




\section{Concluding remarks}

This paper has introduced two new properties, Policy Monotonicity and Intermediateness, which value the goodness of a policy depending on the original profile to which they are applied. This is contrary to the classical tendency that evaluates policies independently of the profile to which they are applied. We have combined these two new properties with three classical properties (Anonymity, Assimilation and Priority for the Poor) in order to construct new criteria to rank opportunity profiles in terms of equality.

One of the key concepts for understanding our properties is the redefinition of the notion of advantage. The literature has studied two main possibilities: the Cardinality-based Criterion and the Partial Inclusion Criterion. We have opted for these two possibilities to construct new criteria to rank opportunity profiles. Clearly, the Partial Inclusion Criterion is the minimal information we may use. Other incomplete criteria could be considered using other information about the desirability of the opportunities. The consideration of such a criterion would change the formulation of some of the properties proposed in this paper, as Policy Monotonicity or Assimilation, and, therefore, the criteria constructed would be different.

Another interesting question for further research would be to study the dynamics of the opportunity profiles across different generations; i.e., mobility. It is generally agreed that the possible inequality present in society is more a cause for concern if the positions of the individuals in the distribution in each generation are exactly the same as the ones that their parents have. On the contrary, inequality is not as bad if the positions in the distribution of the members of the families change across generations (see, for example, Atkinson [4] and Dardanoni [7]). The concept of policy introduced in this paper can be adapted to this dynamic context as the description of the changes from the distribution of opportunities of one generation to the next one. Then, the classification of societies in terms of mobility can be 
performed by ranking policies. However, the analysis of these policies in the mobility framework may be carried out with different axioms that the ones proposed in this paper for measuring equality. For example, imposing that all permutation policies are equivalent independently of the set of individuals that have interchanged their positions, as our Anonymity property establishes, may be controversial depending on the interpretation of mobility adopted. For example, this does not seem to be a good property if we are trying to measure mobility as movement. ${ }^{13}$

\section{Appendix: Proofs of the results}

We will need the following lemmas for the proofs. Let $Q=\left\{\left(x_{1}, x_{2}\right) \in\right.$ $\left.[\mathbb{Q} \cap(0,1)]^{2} \mid x_{1} \leq x_{2} \leq \frac{1-x_{1}}{2}\right\}$.

Lemma 4.1 If $\succsim$ satisfies ANON, ASM, PM-\# and INT, then there exists a complete preorder $R$ over $Q$ such that $O \succsim U$ if and only if $\left(q_{3}(O), q_{2}(O)\right) R$ $\left(q_{3}(U), q_{2}(U)\right)$.

Proof: In Alcalde-Unzu and Ballester [2], we have shown (Lemma 5.6) that a ranking satisfying ANON, ASM and IND-\# can be expressed by a complete preorder $V_{r}$ (with $V_{i}$ and $V_{p}$ as its symmetric and asymmetric parts) over the vectors whose elements are the cardinalities of the individual opportunity sets (henceforth, the domain $T$ ). Here, we do not have IND-\#, but it can be seen that the unique part of this property needed in that result is that in which the two original nested profiles are indifferent. This part is included in PM-\# and, therefore, we have the same result.

We are now going to prove that for all $\vec{x} \in T$ and all $k \in \mathbb{N}, k \vec{x} V_{i} \vec{x}$. Suppose that $\vec{x} V_{p} k \vec{x}$. Then, by INT we have that $\vec{x} V_{p}(k+1) \vec{x} V_{p} k \vec{x}$. Further applications of INT imply that $\vec{x} V_{p}(2 k+1) \vec{x} V_{p} 2 k \vec{x} V_{p} \ldots V_{p}(k+1) \vec{x}$.

\footnotetext{
${ }^{13}$ To see the different approaches to measure mobility, see Van de Gaer et al. [25].
} 
Furthermore, because $(k+1) \vec{x} V_{p} k \vec{x}$, INT implies that $(k+1) \vec{x} V_{p}(2 k+1) \vec{x}$. Hence, by transitivity, $(2 k+1) \vec{x} V_{p}(2 k+1) \vec{x}$, which is not possible. The case of $k \vec{x} V_{p} \vec{x}$ is also impossible and can be proved by similar reasoning. Therefore, the result is straightforward, given that $\sum_{i \in I} q_{i}(O)=1$ for all $O \in L^{3}$.

The following result can be proved using a very similar argument. We omit here the proof.

Lemma 4.2 If $\succsim$ satisfies ANON, ASM, PM- $\subseteq$ and INT, then there exists a complete preorder $R$ over $Q$ such that $O \succsim U$ if and only if $\left(p_{3}(O), p_{2}(O)\right) R$ $\left(p_{3}(U), p_{2}(U)\right)$.

The proof of Theorems 3.1 and 3.2 are from now on equivalent, given that they are based on the equivalence between the criteria on $L^{3}$ and rankings on Q. Additionally, properties PRI and INT have exactly the same implications on $Q$, independently on which of the possible interpretations of the elements of $Q$ (the one deduced in Lemma 4.1 or the one in Lemma 4.2) is selected. Therefore, we opt for including the proof only for Theorem 3.1, but it is easy to see that Theorem 3.2 can be proved following exactly the same steps.

We now introduce the following definitions.

Definition 4.1 For all $\vec{x}, \vec{y} \in Q, \vec{x}$ Lorenz dominates $\vec{y}$ if $x_{1} \geq y_{1}$ and $x_{1}+x_{2} \geq y_{1}+y_{2}$.

Definition 4.2 For all $\vec{x}, \vec{y} \in Q, \vec{x}$ strictly Lorenz dominates $\vec{y}$ if $x_{1}>y_{1}$ and $x_{1}+x_{2}>y_{1}+y_{2}$.

We formulate the following claims that will help us to construct the proof.

Claim 4.1 If $\vec{x}$ Lorenz dominates $\vec{y}$, then $\vec{x} R \vec{y}$, with strict preference if the domination is strict. 
Proof: Let $\vec{x}=\left(\frac{a_{1}}{b_{1}}, \frac{a_{2}}{b_{2}}\right), \vec{y}=\left(\frac{a_{3}}{b_{3}}, \frac{a_{4}}{b_{4}}\right)$ and $t \in \mathbb{N}$ be any common multiple of $\left\{b_{1}, b_{2}, b_{3}, b_{4}\right\}$, where $a_{i}, b_{i} \in \mathbb{N}, i=1, \ldots, 4$. Then, we have $\vec{x}=\left(\frac{n_{1}}{t}, \frac{n_{2}}{t}\right)$ and $\vec{y}=\left(\frac{n_{3}}{t}, \frac{n_{4}}{t}\right)$ for some $n_{1}, n_{2}, n_{3}, n_{4} \in \mathbb{N}$. By assumption, $n_{1} \geq n_{3}$ and $n_{1}+n_{2} \geq n_{3}+n_{4}$. We divide the proof into two cases.

(a) If $n_{2} \geq n_{4}$, we can construct the following chain using PRI:

$$
\left(\frac{n_{1}}{t}, \frac{n_{2}}{t}\right) R\left(\frac{n_{1}-1}{t}, \frac{n_{2}}{t}\right) R \ldots R\left(\frac{n_{3}}{t}, \frac{n_{2}}{t}\right) R\left(\frac{n_{3}}{t}, \frac{n_{2}-1}{t}\right) R \ldots R\left(\frac{n_{3}}{t}, \frac{n_{4}}{t}\right) .
$$

(b) If $n_{4}>n_{2}$, we can construct the following chain using PRI:

$$
\begin{gathered}
\left(\frac{n_{1}}{t}, \frac{n_{2}}{t}\right) R\left(\frac{n_{1}-1}{t}, \frac{n_{2}}{t}\right) R \ldots R\left(\frac{n_{3}+n_{4}-n_{2}}{t}, \frac{n_{2}}{t}\right) R\left(\frac{n_{3}+n_{4}-n_{2}-1}{t}, \frac{n_{2}+1}{t}\right) R \ldots \\
R\left(\frac{n_{3}}{t}, \frac{n_{4}}{t}\right),
\end{gathered}
$$

where use has been made of the assumption that $n_{3}+n_{4}-n_{2} \leq n_{1}$.

The application of transitivity concludes the proof of the weak case.

We now prove the strict case. As above, let $\vec{x}=\left(\frac{a_{1}}{b_{1}}, \frac{a_{2}}{b_{2}}\right)$ and $\vec{y}=\left(\frac{a_{3}}{b_{3}}, \frac{a_{4}}{b_{4}}\right)$. Then, we can rewrite $\vec{x}$ and $\vec{y}$ as $\vec{x}=\left(\frac{n_{1}}{t}, \frac{n_{2}}{t}\right)$ and $\vec{y}=\left(\frac{n_{3}}{t}, \frac{n_{4}}{t}\right)$, with $n_{1}>n_{3}$, $n_{1}+n_{2}>n_{3}+n_{4}$ and $t \in \mathbb{N}$ a common multiple of $\left\{b_{1}, b_{2}, b_{3}, b_{4}\right\}$ for which $\frac{n_{3}+1}{t+1} \leq \frac{n_{1}}{t}$ and $\frac{n_{3}+1}{t+1}+\frac{n_{4}}{t+1} \leq \frac{n_{1}}{t}+\frac{n_{2}}{t}$. Then, we have by the strict part of PRI that $\left(\frac{n_{3}+1}{t+1}, \frac{n_{4}}{t+1}\right) P\left(\frac{n_{3}}{t}, \frac{n_{4}}{t}\right) .{ }^{14}$ Applying the argument in the preceding case, we have that $\left(\frac{n_{1}}{t}, \frac{n_{2}}{t}\right) R\left(\frac{n_{3}+1}{t+1}, \frac{n_{4}}{t+1}\right)$. Transitivity then concludes the proof.

Claim 4.2 For all $\vec{x}, \vec{y} \in Q$ and all $\lambda \in[\mathbb{Q} \cap(0,1)], \vec{x} I \vec{y} \Rightarrow \vec{x} I[\lambda \vec{x}+(1-$ $\lambda) \vec{y}] I \vec{y}$ and $\vec{x} P \vec{y} \Rightarrow \vec{x} P[\lambda \vec{x}+(1-\lambda) \vec{y}] P \vec{y}$.

\footnotetext{
${ }^{14}$ We derived this from PRI starting with a nested profile in which the disadvantaged individual has $n_{3}$ opportunities, the advantaged individual has $t-n_{3}-n_{4}$ opportunities and the individual between them has $n_{4}$ opportunities. Then, the result is obtained by enlarging the opportunity set of the disadvantaged individual by adding an opportunity that is already possessed by the others.
} 
Proof: We prove only the first equivalence as the proof of other parts is similar. Let $\vec{x}=\left(\frac{a_{1}}{b_{1}}, \frac{a_{2}}{b_{2}}\right), \vec{y}=\left(\frac{a_{3}}{b_{3}}, \frac{a_{4}}{b_{4}}\right), \lambda=\frac{\lambda_{1}}{\lambda_{2}} \in(0,1)$ and $t$ be any common multiple of $\left\{b_{1}, b_{2}, b_{3}, b_{4}, \lambda_{2}\right\}$. Consider profiles $O, U \in \mathcal{N}$ such that $O^{\cup} \cap U^{\cup}=\emptyset,\left(\left|O_{3}\right|,\left|O_{2}\right|,\left|O_{1}\right|\right)=\left(n_{1}, n_{2}, t-n_{1}-n_{2}\right)$ and $\left(\left|U_{3}\right|,\left|U_{2}\right|,\left|U_{1}\right|\right)=$ $\left(n_{3}, n_{4}, \frac{1-\lambda}{\lambda} t-n_{3}-n_{4}\right)$, where $n_{1}=\frac{a_{1}}{b_{1}} t, n_{2}=\frac{a_{2}}{b_{2}} t, n_{3}=\frac{a_{3}}{b_{3}} \frac{1-\lambda}{\lambda} t$ and $n_{4}=$ $\frac{a_{4}}{b_{4}} \frac{1-\lambda}{\lambda} t$. Then, we can apply INT and we have that $O \succ(O \cup U) \succ U$. Note that $\left|O_{1}\right|+\left|O_{2}\right|+\left|O_{3}\right|+\left|U_{1}\right|+\left|U_{2}\right|+\left|U_{3}\right|=\frac{t}{\lambda}$. Thus, in terms of $Q,(O \cup U)$ can be expressed as $\left[\left(n_{1}+n_{3}\right) \frac{\lambda}{t},\left(n_{2}+n_{4}\right) \frac{\lambda}{t}\right]=\left[\lambda \frac{a_{1}}{b_{1}}+(1-\lambda) \frac{a_{3}}{b_{3}}, \lambda \frac{a_{2}}{b_{2}}+(1-\lambda) \frac{a_{4}}{b_{4}}\right]$, and we arrive at the desired result.

Claim 4.3 For all $\vec{x}, \vec{y},(k \vec{x}-(k-1) \vec{y}) \in Q$ with $k \in \mathbb{Q}$ such that $k>1$, we have that

$$
\begin{gathered}
\vec{x} I \vec{y} \Leftrightarrow[k \vec{x}-(k-1) \vec{y}] I \vec{x}, \\
\vec{x} P \vec{y} \Leftrightarrow[k \vec{x}-(k-1) \vec{y}] P \vec{x},
\end{gathered}
$$

and

$$
\vec{x} P \vec{y} \Leftrightarrow \vec{y} P[k \vec{y}-(k-1) \vec{x}] .
$$

Proof: We only prove the first equivalence given that the proof of the others is similar. Suppose that $\vec{x} I \vec{y}$, but $[k \vec{x}-(k-1) \vec{y}] P \vec{x}$. Then, $[k \vec{x}-(k-1) \vec{y}] P \vec{y}$. By applying Claim 4.2 to this preference with $\lambda=\frac{1}{k}$, we have that $\vec{x} P \vec{y}$, which is not possible. If, on the other hand, $\vec{x} I \vec{y}$, but $\vec{x} P[k \vec{x}-(k-1) \vec{y}]$, we also have that $\vec{y} P[k \vec{x}-(k-1) \vec{y}]$. Then, we can apply Claim 4.2 with $\lambda=\frac{k-1}{k}$ and we have that $\vec{y} P \vec{x}$, which is not possible.

Claim 4.4 For all $\vec{x}, \vec{y} \in Q$ and all $\lambda_{1}, \lambda_{2} \in \mathbb{Q}$ such that $z_{1}=\lambda_{1} \vec{x}+(1-$ $\left.\lambda_{1}\right) \vec{y}, z_{2}=\lambda_{2} \vec{x}+\left(1-\lambda_{2}\right) \vec{y} \in Q$,

$$
\text { (a) } \vec{x} I \vec{y} \Rightarrow \vec{x} I \overrightarrow{z_{1}} I \overrightarrow{z_{2}} I \vec{y}
$$


and

(b) If $\vec{x} P \vec{y}$, then $\overrightarrow{z_{1}} P \overrightarrow{z_{2}} \Leftrightarrow \lambda_{1}>\lambda_{2}$.

Proof: We prove the second part, and omit the proof of the first part, given that it is proved in a similar way. Suppose that $\overrightarrow{z_{1}} P \overrightarrow{z_{2}}$ but $\lambda_{1} \leq \lambda_{2}$. Reflexivity excludes the possibility that $\lambda_{1}=\lambda_{2}$. Suppose that $\lambda_{1}<\lambda_{2}$. If $\lambda_{2}>0$ and $\lambda_{1}<0$, because $\vec{x} P \vec{y}$, we have $\overrightarrow{z_{2}} P \vec{y}$ by Claim 4.2 and $\vec{y} P \overrightarrow{z_{1}}$ by Claim 4.3. By transitivity, we conclude $\overrightarrow{z_{2}} P \overrightarrow{z_{1}}$, which is not possible. If $\lambda_{i}>0$ for $i=\{1,2\}$, we divide the proof into the following cases:

(a) If $\lambda_{i} \in(0,1)$ for $i=\{1,2\}$, by Claim 4.2 with $\lambda=\lambda_{2}$ we have that $\vec{x} P \overrightarrow{z_{2}} P \vec{y}$. Then, given that $\lambda_{1}<\lambda_{2}$, it follows from $\overrightarrow{z_{2}} P \vec{y}$ and Claim 4.2 that $\overrightarrow{z_{2}} P \overrightarrow{z_{1}} P \vec{y}$, which contradicts the assumption.

(b) If there exists $k \in \mathbb{N}$ such that $\left(\lambda_{i}-k\right) \in(0,1)$ for all $i \in\{1,2\}$, we can deduce from Claim 4.3 that $\vec{x} P \vec{y} \Leftrightarrow[(k+1) \vec{x}-k \vec{y}] P[k \vec{x}-(k-1) \vec{y}]$. Given that $\vec{x} P \vec{y}$, we have that $[(k+1) \vec{x}-k \vec{y}] P[k \vec{x}-(k-1) \vec{y}]$. Now, we can apply Claim 4.2 and we have that $[(k+1) \vec{x}-k \vec{y}] P \overrightarrow{z_{1}} P[k \vec{x}-(k-1) \vec{y}]$. Then, given that $\lambda_{1}<\lambda_{2}$, it follows from $[(k+1) \vec{x}-k \vec{y}] P \overrightarrow{z_{1}}$ and Claim 4.2 that $[(k+1) \vec{x}-k \vec{y}] P \overrightarrow{z_{2}} P \overrightarrow{z_{1}}$, which contradicts the assumption.

(c) If $\lambda_{1} \in(0,1)$ and $\lambda_{2}>1$, we have by Claim 4.3 and INT that $\overrightarrow{z_{2}} P \vec{x}$ and $\vec{x} P \overrightarrow{z_{1}} P \vec{y}$, and applying transitivity we arrive at a contradiction.

(d) If $\left(\lambda_{1}-k\right) \in(0,1)$ and $\left(\lambda_{2}-k^{\prime}\right) \in(0,1)$ with $k<k^{\prime}$, we can apply reasoning similar to that used in the preceding case.

The case in which $\lambda_{i}<0$ is similar and, thus, it is omitted.

Claim 4.5 For all $\vec{\alpha} \in \mathbb{Z}^{2}$ and all $\vec{x}, \vec{y},(\vec{x}+\vec{\alpha}),(\vec{y}+\vec{\alpha}) \in Q$, we have that

$$
(\vec{x}+\vec{\alpha}) R \vec{x} \Leftrightarrow(\vec{y}+\vec{\alpha}) R \vec{y} .
$$


Proof: First, we know from Claim 4.1 that for all $\vec{\alpha} \in \mathbb{Z}^{2}$ such that $\alpha_{1}>0$ and $\alpha_{1}+\alpha_{2}>0$ that $(\vec{x}+\vec{\alpha}) P \vec{x}$ for all $\vec{x} \in Q$. We can also deduce from Claim 4.1 that for all $\vec{\alpha} \in \mathbb{Z}^{2}$ such that $\alpha_{1}<0$ and $\alpha_{1}+\alpha_{2}<0$, we have that $\vec{x} P(\vec{x}+\vec{\alpha})$ for all $\vec{x} \in Q$. Thus, without loss of generality, we only need to consider the cases in which $\alpha_{1} \geq 0, \alpha_{2}<0$ and $\alpha_{1} \leq\left|\alpha_{2}\right|{ }^{15}$ We divide the proof into the following cases:

(a) If $\vec{x}$ Lorenz dominates $\vec{y}$, we have by Claim 4.1 that $\vec{x} R \vec{y}$. Suppose that $(\vec{x}+\vec{\alpha}) R \vec{x}$. Then we can apply PM-\# and we have that $(\vec{y}+\vec{\alpha}) R \vec{y}$. Now, suppose that $\vec{x} R(\vec{x}+\vec{\alpha})$. We also know that $(\vec{x}+\vec{\alpha})$ Lorenz dominates $(\vec{y}+\vec{\alpha})$. Then we can apply PM-\# and we have that $\vec{y} R(\vec{y}+\vec{\alpha})$.

(b) If $\vec{y}$ Lorenz dominates $\vec{x}$, the reasoning is similar to that used in part (a).

(c) If there is no Lorenz domination between $\vec{x}$ and $\vec{y}$, suppose, without loss of generality, that $y_{1}>x_{1}, y_{1}+y_{2}<x_{1}+x_{2}$ and $(\vec{x}+\vec{\alpha}) R \vec{x}$. Then, we have that $y_{1}=x_{1}+\lambda_{1} \alpha_{1}$ and $y_{2}=x_{2}+\lambda_{2} \alpha_{2}$. If $\lambda_{1}=\lambda_{2}=\lambda>0$, we know that $\vec{y}=\vec{x}+\lambda \vec{\alpha}$ and $(\vec{y}+\vec{\alpha})=\vec{x}+(\lambda+1) \vec{\alpha}$. Then, with $k=\lambda+1$, we have that $[k(\vec{y}+\vec{\alpha})-(k-1) \vec{x}]=\vec{y}$. Given that $(\vec{x}+\vec{\alpha}) R \vec{x}$, we can apply Claim 4.3 and we have that $(\vec{y}+\vec{\alpha}) R \vec{y}$. If $\lambda<0$, we can prove this claim by reduction to the absurd. Suppose that $\vec{y} P(\vec{y}+\vec{\alpha})$. Then, we can deduce by the same reasoning as above that $\vec{x} P(\vec{x}+\vec{\alpha})$, which leads us to a contradiction.

If $\lambda_{1}<\lambda_{2}$, consider the vectors $\vec{z}=\left(y_{1}, x_{2}+\lambda_{1} \alpha_{2}\right), \vec{w}=\left(y_{1}+\alpha_{1}, x_{2}+\right.$ $\left.\left(\lambda_{1}+1\right) \alpha_{2}\right) \in Q$, that obviously exist. We have that $\vec{z}$ and $\vec{w}$ satisfies the conditions of the above case. Therefore, $\vec{w} R \vec{z}$. We also have by Claim 4.1 that $\vec{z} R \vec{y}$. Then, we can apply PM-\# and we have that $(\vec{y}+\vec{\alpha}) R \vec{y}$. The case in which $\lambda_{1}>\lambda_{2}$ can be proved by similar reasoning.

\footnotetext{
${ }^{15}$ The remaining cases can be easily deduced from this analysis.
} 
In view of the preceding results, we can focus exclusively on the changes in the percentages of the total number of opportunities available to the individuals. We define a correspondence $g:\left(\mathbb{R}_{+} \cup\{\infty\}\right) \rightarrow\{G, B\}$ in the following way:

$$
\begin{aligned}
& G \in g(x) \Leftrightarrow(\vec{y}+\vec{\alpha}) R \vec{y} \text { for all } \vec{y},(\vec{y}+\vec{\alpha}) \in Q \text { for which } \frac{\alpha_{1}}{\alpha_{2}}=x . \\
& B \in g(x) \Leftrightarrow \vec{y} R(\vec{y}+\vec{\alpha}) \text { for all } \vec{y},(\vec{y}+\vec{\alpha}) \in Q \text { for which } \frac{\alpha_{1}}{\alpha_{2}}=x .
\end{aligned}
$$

Claim 4.6 If $x, y \in\left(\mathbb{R}_{+} \cup\{\infty\}\right)$ such that $x>y$, then

$$
\begin{aligned}
& G \in g(y) \Rightarrow G=g(x) \\
& B \in g(x) \Rightarrow B=g(y)
\end{aligned}
$$

Proof: We prove only the first part of the claim, the proof of the second part is similar. Suppose that $G \in g(y)$, with $\frac{\alpha_{1}}{\alpha_{2}}=y$. We also know that $\frac{\beta_{1}}{\beta_{2}}=x$. Then, we can rewrite $x$ and $y$ as $\frac{\alpha_{1} \beta_{2}}{\alpha_{2} \beta_{2}}=y$ and $\frac{\beta_{1} \alpha_{2}}{\alpha_{2} \beta_{2}}=x$. As $g \in G(y)$, we know that $\left(y_{1}+\alpha_{1} \beta_{2}, y_{2}+\alpha_{2} \beta_{2}\right) R \vec{z}$ for all $\vec{z},\left(z_{1}+\alpha_{1} \beta_{2}, z_{2}+\right.$ $\left.\alpha_{2} \beta_{2}\right) \in Q$. Given that $\beta_{1} \alpha_{2}>\alpha_{1} \beta_{2}$, we can apply Claim 4.1 and we have that $\left(z_{1}+\beta_{1} \alpha_{2}, z_{2}+\alpha_{2} \beta_{2}\right) P\left(z_{1}+\alpha_{1} \beta_{2}, z_{2}+\alpha_{2} \beta_{2}\right)$ and by transitivity, that $\left(z_{1}+\beta_{1} \alpha_{2}, z_{2}+\alpha_{2} \beta_{2}\right) P \vec{z}$. Therefore, $G=g(x)$.

We now define

$$
x_{*}=\inf \left[x \in\left(\mathbb{R}_{+} \cup\{\infty\}\right) \text { such that } G \in g(x)\right]
$$

and

$$
x^{*}=\sup \left[x \in\left(\mathbb{R}_{+} \cup\{\infty\}\right) \text { such that } B \in g(x)\right] .
$$

We are going to prove that $x_{*}=x^{*}$. If $x_{*}>x^{*}$, there exists $y \in \mathbb{R}$ such that $x_{*}>y>x^{*}$. That is, $G \notin g(y)$ and $B \notin g(y)$, which is not possible. If $x_{*}<x^{*}$, then there exists $z \in \mathbb{R}$ such that $x_{*}<z<x^{*}$. Then, we have 
by Claim 4.6 that $g(z)=G$ and $g(z)=B$, which is not possible. Therefore, $x_{*}=x^{*}=\beta$.

It is now straightforward to see that the rankings are such that

$$
x_{1}+\alpha x_{2}>y_{1}+\alpha y_{2} \Rightarrow \vec{x} P \vec{y}
$$

Furthermore, PRI ensures that $\alpha \in[0,1]$, and Theorem 3.1 is proved.

\section{Independence of the axioms}

Consider the following rankings:

$$
\begin{gathered}
O \succsim_{a} U \Leftrightarrow \frac{\left|O_{3}\right|}{C S(O)} \geq \frac{\left|U_{3}\right|}{C S(U)} \\
O \succsim_{b} U \Leftrightarrow q_{3}(O)-2 \cdot q_{1}(O) \geq q_{3}(U)-2 \cdot q_{1}(U) \\
O \sim_{c} U \text { for all } O, U \in L^{3} \\
O \succsim_{d} U \Leftrightarrow p_{3}(O) \geq p_{3}(U) \\
O \succsim_{e} U \Leftrightarrow\left|O_{\sigma(3)} \geq\right| U_{\sigma(3)} \mid \\
O \succsim_{f} U \Leftrightarrow q_{3}(O)>q_{3}(U) \text { or }\left[q_{3}(O)=q_{3}(U) \text { and } q_{2}(O)>q_{2}(U)\right] \\
O \succsim_{g} U \Leftrightarrow \frac{|O \cap|+\left|O_{2} \cap O O_{3}\right|}{C S(O)} \geq \frac{\left|U^{\cap}\right|+\left|U_{2} \cap U_{3}\right|}{C S(U)} \\
\Leftrightarrow p_{3}(O)-2 \cdot p_{1}(O) \geq p_{3}(U)-2 \cdot p_{1}(U) \\
O{ }^{n}(U)
\end{gathered}
$$




$$
\begin{gathered}
O \succsim_{i} U \Leftrightarrow q_{3}(O) \geq q_{3}(U) \\
O \succsim_{j} U \Leftrightarrow\left|O_{\cap}\right| \geq\left|U_{\cap}\right|
\end{gathered}
$$

$$
O \succsim_{k} U \Leftrightarrow p_{3}(O)>p_{3}(U) \text { or }\left[p_{3}(O)=p_{3}(U) \text { and } p_{2}(O)>p_{2}(U)\right]
$$

It is easy to see that $\succsim_{a}$ satisfy all axioms of Corollary 3.1 except ANON. Similarly, $\succsim_{b}$ satisfies all axioms except ASM, $\succsim_{c}$ satisfies all properties except PRI and $\succsim_{d}$ satisfies all axioms except PM-\#. Finally, $\succsim_{e}$ satisfies all axioms except INT and $\succsim_{f}$ satisfy all properties except ARCHD.

Similarly, $\succsim g$ satisfy all axioms of Corollary 3.2 except ANON. Similarly, $\succsim_{h}$ satisfies all axioms except $\mathrm{ASM}, \succsim_{c}$ satisfies all properties except PRI and $\succsim_{i}$ satisfies all axioms except PM- $\subseteq$. Finally, $\succsim_{j}$ satisfies all axioms except INT and $\succsim_{f}$ satisfy all properties except ARCHD.

\section{References}

[1] J. Alcalde-Unzu, R. Arlegi and J. Nieto, Cardinality-based Equality of opportunities, Review of Economic Design, 10 (2007), 285-304.

[2] J. Alcalde-Unzu and M. Ballester, On the axiomatic distribution of opportunities, Social Choice and Welfare 34 (2010), 3-31.

[3] R. Arneson, Equality and equality of opportunity for welfare, Philosophical Studies, 56 (1989), 77-93.

[4] A. B. Atkinson, Social Justice and Public Policy, The M.I.T. Press, (1983). 
[5] S. Barbera and M. Jackson, Maximin, Leximin and the Protective Criterion: characterizations and comparisons, Journal of Economic Theory 46 (1988), 34-44.

[6] W. Bossert, M. Fleuerbaey and D. van de Gaer, Responsibility, talent, and compensation: A second-best analysis, Review of Economic Design 4 (1999), 35-55.

[7] V. Dardanoni, Measuring social mobility, Journal of Economic Theory 61 (1993), 372-394.

[8] R. Dworkin, What is Equality? Part 1: Equality of Welfare, Philosophy and Public Affairs 10 (1981), 185-246.

[9] R. Dworkin, What is Equality? Part 1: Equality of Resources, Philosophy and Public Affairs 10 (1981), 283-345.

[10] R. Echávarri and I. Permanyer, Ranking profiles of capability sets, Social Choice and Welfare 31 (2008), 521-535

[11] J. Elster, Sour grapes-utilitarianism and the genesis of wants. In: Sen AK, Williams B (eds) Utilitarianism and beyond. Cambridge University Press, Cambridge, (1982).

[12] M. Fleurbaey, Living standards and capabilities: Equal values or equal sets?, Analyse $\&$ Kritik 29 (2007), 226-234.

[13] C. Herrero, Equitable opportunities: An extension, Economic Letters 55 (1997), 91-95.

[14] C. Herrero, I. Iturbe-Ormaetxe and J.Nieto, Ranking opportunity profiles on the basis of the common opportunities, Mathematical Social Sciences 35 (1998), 273-289.

[15] M. King, An index of inequality: With applications to horizontal equity and social mobility, Econometrica 51 (1983), 99-115. 
[16] L. Kranich, Equitable opportunities: An axiomatic approach, Journal of Economic Theory 71 (1996), 131-147.

[17] E. A. Ok, On opportunity inequality measurement, Journal of Economic Theory $\mathbf{7 7}$ (1997), 300-329.

[18] E. A. Ok and L. Kranich, The measurement of opportunity inequality: A cardinality-based approach, Social Choice and Welfare 15 (1998), 263-287.

[19] P. Pattanaik and Y. Xu, On ranking opportunity sets in terms of freedom of choice, Recherches Economiques de Louvain 56, (1990), 383-390

[20] V. Peragine, The distribution and the redistribution of opportunities, Journal of Economic Surveys 13 (1999), 37-69.

[21] J. Rawls, A Theory of Justice, Harvard University Press, Cambridge, Massachusetts, (1971).

[22] E. Savaglio and S. Vanucci, Filtral preorders and opportunity inequality, Journal of Economic Theory 132 (2007), 474-492.

[23] A.K. Sen, Commodities and Capabilities, North-Holland, Amsterdam, (1985).

[24] A.K. Sen, Welfare, preference and freedom, Journal of Econometrics 50 (1991), 15-29.

[25] D. Van de Gaer, E. Schokkaert and M. Martinez, Three Meanings of Intergenerational Mobility, Economica 68 (2001), 519-537.

[26] J. Weymark, Generalized Gini indices of equality of opportunity, Journal of Economic Inequality 1 (2003), 5-24. 